\title{
Genetics of larval urea tolerance in Drosophila melanogaster
}

\author{
AMITABH JOSHI, CHRISTOPHER D. KNIGHT \& LAURENCE D. MUELLER* \\ Department of Ecology and Evolutionary Biology, University of California, Irvine, CA 92717, U.S.A.
}

\begin{abstract}
The genetic control of larval tolerance to urea, a nitrogenous waste-product occurring naturally in crowded Drosophila cultures, was investigated in a set of five laboratory populations of $D$. melanogaster that had been successfully subjected to selection for increased larval urea tolerance. Larva to adult survivorship and development time at three different levels of urea were assayed on the five selected populations, their five matched controls and a set of $10 \mathrm{~F}_{1}$ hybrid populations derived from reciprocal crosses between pairs of selected and control populations. As expected from the results of previous studies, the selected populations exhibited greater larval tolerance to the toxic effects of urea, relative to their controls. Comparison of the hybrid and parental populations with respect to both survivorship and development time indicated that the genetic control of urea tolerance in the selected populations is largely dominant, and has a significant $\mathrm{X}$-linked component. The data also suggested that females from the selected populations exercise a nongenetic maternal effect on the development time of their progeny, regardless of urea level.
\end{abstract}

Keywords: density-dependent selection, Drosophila melanogaster, larval crowding, stress resistance, urea tolerance, X-linkage.

\section{Introduction}

In the decades following the seminal work of MacArthur \& Wilson (1967), much progress has been made in understanding how density-dependent natural selection may act in moulding the evolution of life histories. Most of our understanding of the biological details of this important phenomenon has come from empirical studies using species of Drosophila as a model system (reviewed by Mueller, 1995). Initially, such studies focused on understanding the biology of traits either known, or thought, to be advantageous under conditions of extreme larval crowding. These traits ranged from primarily behavioural attributes such as pupation height (Sameoto \& Miller, 1968; Markow, 1979), larval feeding rate (Burnet et al., 1977) and the extent of larval locomotory activity while feeding (Sokolowski, 1985; Godoy-Herrera, 1986) to those that were predominantly physiological, such as larval efficiency at converting food to biomass (de Miranda \& Eggleston, 1988) and larval tolerance to nitrogenous metabolic waste (Botella et al., 1985). Subsequently, the relevance of many of these traits to the density-

\footnotetext{
${ }^{*}$ Correspondence.
}

(C) 1996 The Genetical Society of Great Britain. dependent evolution of life histories was explicitly demonstrated by showing that these traits did, in fact, evolve in laboratory populations of $D$. melanogaster maintained at high larval densities for many generations (Mueller \& Sweet, 1986; Joshi \& Mueller, 1988, 1993, 1996; Mueller, 1990; Mueller et al., 1991, 1993).

Many of these traits, especially those related to larval behaviour, are also fairly well understood at the genetic level, adding substantially to our appreciation of their role in the density-dependent evolution of Drosophila life history strategies (Sewell et al., 1975; Bauer \& Sokolowski, 1985, 1988; de Belle \& Sokolowski, 1987; de Belle et al., 1989, 1993; Godoy-Herrera, 1994; Pereira et al., 1995). In contrast to these behavioural traits, little is known about the genetic control of larval tolerance to the toxic effects of urea, a potentially significant component of adaptation to high larval densities in Drosophila (Mueller, 1995), beyond the fact that successful selection for increased larval urea tolerance is possible (J. Shiotsugu, A. M. Leroi, H. Yashiro, M. R. Rose \& L. D. Mueller, unpublished data). In studies of interspecific competition between Drosophila species, the inhibitory effects of larval biotic residues on both larval viability (Budnick \& Brncic, 
1974, 1976) and female fecundity (Aiken \& Gibo, 1979) were observed. Subsequently, exposure to urea was shown to result in the slower development and reduced survivorship of $D$. melanogaster larvae (Botella et al., 1983, 1985). In our laboratory, we have also observed an inhibition of fecundity in female $D$. melanogaster maintained as adults on urea-supplemented food (A. Joshi, J. Shiotsuga \& L. D. Mueller, unpublished data).

Although it is not the primary metabolic waste of Drosophila larvae, urea has been shown to accumulate in Drosophila culture vials during the larval phase (Botella et al., 1985). In our laboratory, we have observed that the rate at which nitrogenous waste accumulates in the food medium is much higher in crowded cultures, although it is not clear at this time whether the principal form of nitrogenous waste building up in these cultures is urea or ammonia, because the technique used converts urea to ammonia in order to assay urea level (A. G. Gibbs, D. J. Borash \& L. D. Mueller, unpublished data). Not surprisingly, larvae from populations of D. melanogaster maintained in our laboratory at high larval densities for many generations, have also been seen to be more tolerant to the detrimental effects of urea on various fitness components, relative to their uncrowded controls (J. Shiotsugu et al., unpublished data).

In this paper, we report results from an experiment in which we investigated the genetic control of urea tolerance in a set of five large, outbred, laboratory populations of $D$. melanogaster that had been subjected to direct selection for increased larval tolerance to urea. These populations had earlier been seen to have diverged from their controls in several measures of urea tolerance, including egg to adult survival and development time when raised as larvae on urea-supplemented food (J. Shiotsugu et al., unpublished data). These selected lines were subjected to levels of urea in excess of those normally occurring in crowded cultures in order to promote their rapid, and extensive, differentiation from the control lines, with regard to larval urea tolerance; such highly differentiated lines provide the degree of resolution needed for the genetic analysis of quantitative traits.

\section{Materials and methods}

\section{Experimental populations}

This study used five populations of $D$. melanogaster that have been subjected to selection for increased larval tolerance to the presence of toxic levels of urea in the food, as well as five control populations. The five populations selected for increased larval tolerance to urea $\left(\mathrm{MX}_{1}-\mathrm{MX}_{5}\right)$ are raised as larvae at low densities (50-100 eggs per 8 dram vial) on banana-molasses food with urea added to it. Adult flies are allowed to oviposit on non-nutritive agar for approximately $6 \mathrm{~h}$; the eggs are then placed into the food vials at the appropriate density. The following day, plastic sleeves are inserted into each vial. Once most larvae have pupated onto the plastic inserts, these are removed and placed into cages with Petri dishes containing yeasted banana-molasses food. This procedure ensures that adult flies will not be exposed to urea, so as to avoid confounding the results of adaptation to larval and adult exposure to environmental urea. Food is changed daily for 5 to 6 days after peak eclosion until egg collection for the next generation takes place. The five control populations $\left(\mathrm{MC}_{1}-\mathrm{MC}_{5}\right)$ are maintained on an identical regime except that they are reared on regular banana-molasses food as larvae. The generation time of both MX and MC populations is about 3 weeks, and all populations are maintained at $25^{\circ} \mathrm{C}$ on a 24 h light regime.

Both sets of populations were derived from the five B populations of Rose (1984), each B population being used as the progenitor of one $\mathrm{MX}$ and one $\mathrm{MC}$ population $\left(\mathrm{MX}_{i}, \mathrm{MC}_{i}\right.$ derived from $\mathrm{B}_{i}$, $i=1-5)$. Consequently, $\mathrm{MX}$ and $\mathrm{MC}$ populations bearing the same numerical subscript are more closely related to each other, compared with other populations subjected to the same selection regime. Initially, the MX populations were reared on $12 \mathrm{~g}$ $\mathrm{L}^{-1}$ urea in the food; this was increased to $14 \mathrm{~g} \mathrm{~L}^{-1}$ at generation 5, and then further increased to its current concentration of $18 \mathrm{~g} \mathrm{~L}^{-1}$ at generation 25 . At the time of this study, the MX and MC populations had been maintained in the laboratory for approximately 65 generations.

\section{Hybrid populations}

To eliminate any environmental or parental effects, all experimental populations were subjected to two generations under identical rearing conditions, before setting up the crosses from which the $F_{1}$ hybrids were obtained. In the third generation, virgin adults from each population were collected every $6 \mathrm{~h}$. Adult males and females were kept separate and used to set up homotypic and heterotypic crosses. For example, adult male and female virgins from populations $M X_{1}$ and $M_{1}$ were used in four distinct crosses as follows (the first letter in a hybrid population name identifies the population that 
supplied the male parents).

(a) Homotypic crosses:

(i) male $\mathrm{MC}_{1} \times$ female $\mathrm{MC}_{1} \rightarrow$ population $\mathrm{MC}_{1}$ (parental);

(ii) male $\mathrm{MX}_{1} \times$ female $\mathrm{MX}_{1} \rightarrow$ population $\mathrm{MX}_{1}$ (parental).

(b) Heterotypic crosses:

(i) male $\mathrm{MC}_{1} \times$ female $\mathrm{MX}_{1} \rightarrow$ population $\mathrm{CX}_{1}$ (hybrid);

(ii) male $\mathrm{MX}_{1} \times$ female $\mathrm{MC}_{1} \rightarrow$ population $\mathrm{XC}_{1}$ (hybrid).

Similar sets of four crosses were carried out for each of the other pairs of MX and MC populations; a total of 20 populations was thus assayed in this study.

\section{Measurement of urea tolerance}

To assay urea tolerance, freshly hatched first instar larvae from each of the above crosses were collected. Egg-hatch to adult survivorship and development time were measured at three different levels of urea: 0,14 and $18 \mathrm{~g}$ urea $\mathrm{L}^{-1}$ of bananamolasses food. Five vials, each with 40 larvae, were set up at each level of urea for each individual population. Eclosing adults were collected every $6 \mathrm{~h}$ and the number of males and females recorded. These data were subsequently used to calculate egg-hatch to adult development time and survivorship.

\section{Statistical analysis}

All statistical analyses were performed using SAS for Windows version 6.08. Analyses of variance (ANOVA) were used to investigate differences in the survivorship and development time between the parental and hybrid populations. The survivorship data were transformed using the arcsine-square root transformation (Freeman \& Tukey, 1950); analyses for development time were performed on both untransformed and log-transformed data. The results of the analysis were not qualitatively affected by log-transformation and, consequently, the results presented here are those from the analysis of the untransformed data. The data were analysed in a completely randomized block design, because of the pattern of relatedness between MX and MC populations, with population (specific parent or hybrid), and urea level being treated as fixed factors crossed with the different blocks (replicates 1-5) representing the random effect of ancestry. For survivorship data, the units of analysis were the fraction of larvae surviving to eclosion per vial. Mean survivorship of both the $\mathrm{CX}$ and $\mathrm{XC}$ hybrids was compared to the mid-parent value in a separate ANOVA, using Scheffé's test for multiple comparisons; this test was performed on the means of individual replicate populations and the family type I error rate was controlled at 0.05 . For the development time data, measurements on individual flies were used as units of analysis; vial was treated as a random factor nested within the block $\times$ population $\times$ urea interaction, and sex was treated as a fixed factor crossed with all the rest.

\section{Results}

\section{Egg-hatch to adu/t survivorship}

The results showed a clear superiority of the MX populations over the $\mathrm{MC}$ and the hybrid populations in the presence of urea in the food; in the absence of urea there were no significant differences among any of the populations (Fig. 1). This patiern was reflected in the significant effects of population and

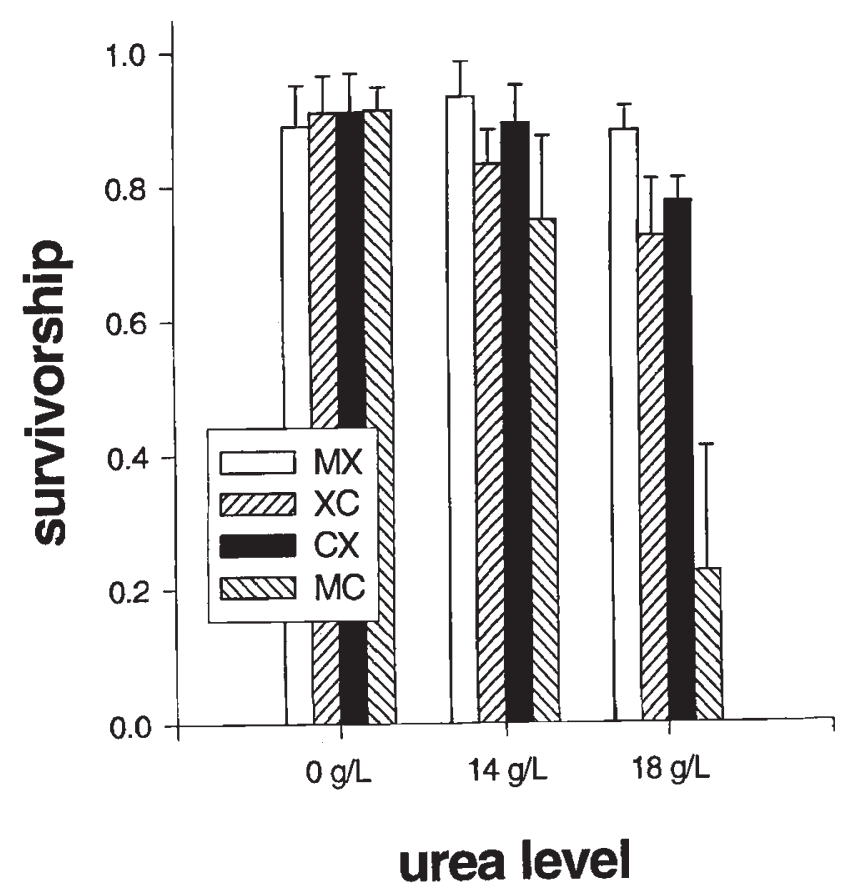

Fig. 1 Egg-hatch to adult survivorship in the parental and hybrid populations of Drosophila melanogaster at three different levels of urea. Error bars represent $95 \%$ confidence intervals about the mean of five replicate populations and are based on the variance between replicate populations. As the analyses of variance (ANOVA) were performed using a block design, and on transformed data, these confidence intervals, that are meant for indicating the range of variation, should not be used for visual hypothesis testing. 
Table 1 Results of analysis of variance (ANOVA) on egg-hatch to adult survivorship in the MX and MC populations of Drosophila melanogaster, and their hybrids

\begin{tabular}{lrrrc}
\hline Source & d.f. & MS & $F$ & $P$ \\
\hline Block & 4 & 0.1149 & 4.94 & $<0.001$ \\
Population & $\mathbf{3}$ & $\mathbf{1 . 7 2 4 3}$ & $\mathbf{2 8 . 1 0}$ & $<\mathbf{0 . 0 0 0 5}$ \\
Urea & $\mathbf{2}$ & $\mathbf{3 . 0 6 7 8}$ & $\mathbf{2 3 0 . 7 9}$ & $<\mathbf{0 . 0 0 0 5}$ \\
Block $\times$ population & 12 & 0.0614 & 2.64 & $<0.005$ \\
Block $\times$ urea & 8 & 0.0133 & 0.57 & $>0.80$ \\
Population $\times$ urea & $\mathbf{6}$ & $\mathbf{0 . 8 2 9 1}$ & $\mathbf{2 1 . 0 3}$ & $<\mathbf{0 . 0 0 1}$ \\
Block $\times$ population $\times$ urea & 24 & 0.0394 & 1.69 & $<0.05$ \\
Error & 240 & 0.0233 & & \\
\hline Significant & & & & \\
\hline
\end{tabular}

Significant fixed main effects and interactions are indicated in boldface.

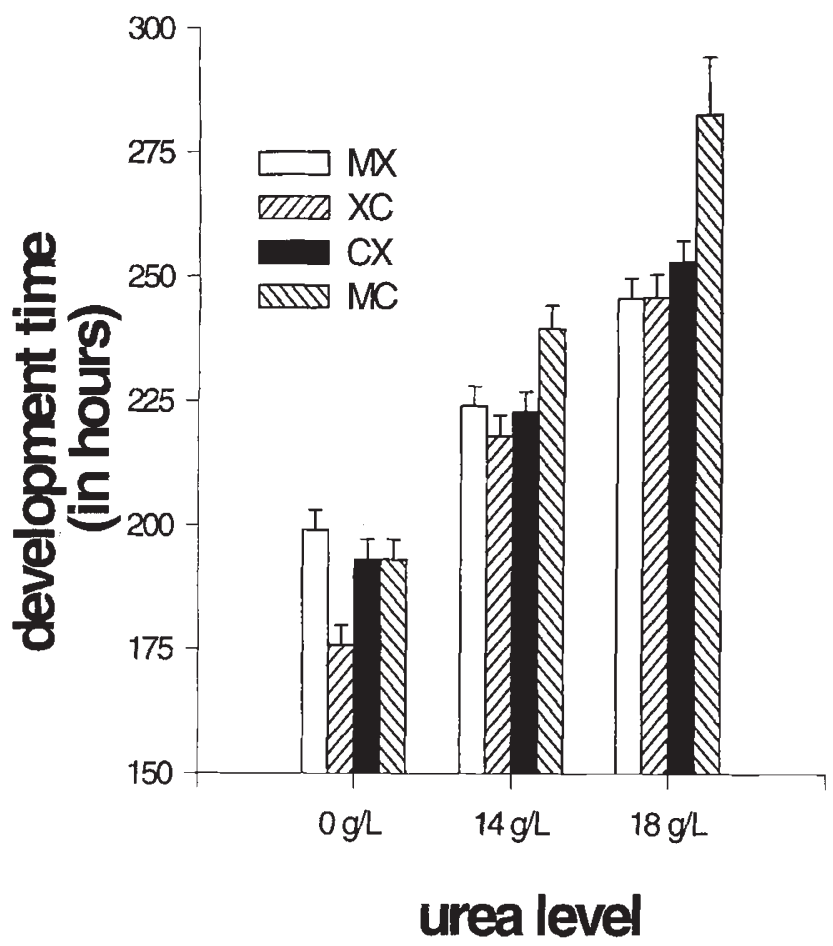

Fig. 2 Development time (egg-hatch to eclosion) in the parental and hybrid populations of Drosophila melanogaster at three different levels of urea. Error bars represent $95 \%$ confidence intervals about the mean of five replicate populations, and were calculated using least squares estimates of the standard errors of cell means in the randomized block ANOVA.

the population $\times$ urea interaction in the ANOVA (Table 1). Both hybrid populations exhibited survivorship significantly greater than the mid-parent value $(P<0.005)$, indicating a substantial degree of dominance for larval tolerance to urea. Multiple comparisons suggested that the $\mathrm{CX}$ hybrids may have superior suvivorship on urea compared with the XC hybrids. Although the difference between the two types of hybrid was not significant in the overall ANOVA $(P=0.19)$, the two differed significantly when compared with one another, and the mid-parent values in a separate ANova $(P=0.01)$. Moreover, mean survivorship of $\mathrm{CX}$ populations was not significantly different from that of the MX parents at $14 \mathrm{~g} \mathrm{~L}^{-1}$, or averaged across all levels of urea. The XC hybrids, on the other hand, showed significantly lower survivorship than the MX parents both at $14 \mathrm{~g}$ urea $\mathrm{L}^{-1}$, and overall. At $14 \mathrm{~g}$ urea $\mathrm{L}^{-1}$, suvivorship of the CX populations was almost significantly greater than that of the $\mathrm{XC}$ populations $(P=0.0545)$. At the $18 \mathrm{~g}$ urea $\mathrm{L}^{-1}$ level, the $\mathrm{CX}$ populations showed significantly lower survivorship than the MX parents. Their survivorship was still greater than that of the XC hybrids, although the difference was not significant.

\section{Egg-hatch to eclosion development time}

Once again, the ANOVA results revealed significant effects of population and the population $\times$ urea interaction (Table 2). As in the case of survivorship, the data showed superiority of the MX populations in the presence of urea (Fig. 2). As the urea level increased from 0 to $18 \mathrm{~g}$ urea $\mathrm{L}^{-1}$, the development time of the MX populations changed from being the greatest (slowest) to the least (quickest); at $0 \mathrm{~g} \mathrm{~L}^{-1}$, the MX populations had significantly slower development than the MC populations $(P=0.008)$, probably indicative of a cost of maintaining tolerance to urea. The marked increase in development time of the MC populations with increasing levels of urea represents the detrimental effect of urea; increased development time is an indication of decreased fitness. Overall, the XC hybrids developed significantly faster than the $\mathrm{CX}$ hybrids $(P<0.05)$, 
Table 2 Results of analysis of variance (ANOVA) on egg-hatch to eclosion development time in the MX and MC populations of Drosophila melanogaster, and their hybrids

\begin{tabular}{lrrrc}
\hline Source & d.f. & \multicolumn{1}{c}{ MS } & \multicolumn{1}{c}{$\boldsymbol{F}$} & $P$ \\
\hline Block & 4 & 4753 & 5.91 & $<0.001$ \\
Population & $\mathbf{3}$ & $\mathbf{1 3 2 3 7 8}$ & $\mathbf{5 . 2 4}$ & $<\mathbf{0 . 0 2 5}$ \\
Urea & $\mathbf{2}$ & $\mathbf{2 3 4 5 6 7 7}$ & $\mathbf{1 6 6 0 . 0 7}$ & $<\mathbf{0 . 0 0 1}$ \\
Sex & $\mathbf{1}$ & $\mathbf{1 0 9 5 1 6}$ & $\mathbf{2 4 3 . 2 0}$ & $<\mathbf{0 . 0 0 1}$ \\
Vial $($ block $\times$ population $\times$ urea) & 239 & 803 & 3.40 & $<0.001$ \\
Block $\times$ population & 12 & 25259 & 31.45 & $<0.001$ \\
Block $\times$ urea & 8 & 1414 & 1.76 & $>0.05$ \\
Block $\times$ sex & 4 & 450 & 1.91 & $>0.05$ \\
Population $\times$ urea & $\mathbf{6}$ & $\mathbf{4 2 4 9 7}$ & $\mathbf{2 2 . 6 8}$ & $<\mathbf{0 . 0 0 1}$ \\
Population $\times$ sex & $\mathbf{3}$ & $\mathbf{2 8 6 1}$ & $\mathbf{5 . 3 8}$ & $<\mathbf{0 . 0 2 5}$ \\
Urea $\times$ sex & 2 & 1083 & 2.80 & $>0.05$ \\
Sex $\times$ vial $($ block $\times$ population $\times$ urea) & 236 & 236 & 1.00 & $>0.05$ \\
Block $\times$ population $\times$ urea & 24 & 1874 & 2.34 & $<0.001$ \\
Block $\times$ population $\times$ sex & 12 & 532 & 2.25 & $<0.025$ \\
Block $\times$ urea $\times$ sex & 8 & 387 & 1.64 & $>0.05$ \\
Population $\times$ urea $\times$ sex & $\mathbf{6}$ & $\mathbf{1 2 7 9}$ & $\mathbf{3 . 9 7}$ & $<\mathbf{0 . 0 1}$ \\
Block $\times$ population $\times$ urea $\times$ sex & 24 & 322 & 1.37 & $>0.05$ \\
Error & 9209 & 236 & & \\
\hline
\end{tabular}

Nesting of effects is indicated by parentheses. Significant fixed main effects and interactions are shown in boldface.

but this difference became less pronounced in the presence of urea (Fig. 2). The faster development of the XC hybrids, compared with their CX counterparts, suggests a maternal effect whereby individuals with an MX mother develop relatively slowly, even in the absence of urea. In the presence of urea, both hybrids showed development times similar to the MX parents, and significantly greater than the MC parents, again suggesting a substantial dominance component to the larval urea tolerance of the MX populations.

The ANOVA also showed a significant population $\times$ urea $\times$ sex interaction, and multiple comparisons among cell means of the various populatio$\mathrm{n} \times$ urea $\times$ sex combinations provided further evidence for an X-linked component to urea tolerance in the MX populations. Under the assumption of dominance, if urea tolerance were $\mathrm{X}$-linked, the urea tolerance of $\mathrm{XC}$ and $\mathrm{CX}$ females would be expected to be similar. However, CX males, inheriting their $\mathrm{X}$ chromosome from their MX mothers, would be expected to show greater urea tolerance than XC males, because the latter would inherit their $\mathrm{X}$ chromosome from their maternal $\mathrm{MC}$ parents. The results show that the development time of $\mathrm{CX}$ females, relative to $\mathrm{XC}$ females, remained significantly greater across all urea levels (Fig. 3). As
$\mathrm{CX}$ and $\mathrm{XC}$ hybrids do not differ in the relative contribution of the MX parent to their genome, this result supports the notion of a detrimental maternal effect on the development time of the offspring of MX mothers. The performance of CX males, relative to XC males, however, improved as urea levels increased. At $0 \mathrm{~g}$ urea $\mathrm{L}^{-1}$, the $\mathrm{CX}$ males had significantly greater development time than $\mathrm{XC}$ males. This difference diminished at $14 \mathrm{~g}$ urea $\mathrm{L}^{-1}$, and was not seen at all at $18 \mathrm{~g}$ urea $\mathrm{L}^{-1}$ (Fig. 3), suggesting that in the presence of urea the greater urea tolerance of the CX males was enough to compensate completely for their inherently greater development time.

\section{Discussion}

The superior larval urea tolerance of the MX populations, relative to the $\mathrm{MC}$ controls, seen in this study, confirms the preliminary observation of a successful response to selection for increased urea tolerance in the MX populations (J. Shiotsugu et al., unpublished data). Moreover, the results of the present study extend the preliminary work of Shiotsugu et al. by providing information about the genetic control of larval urea tolerance in these populations. Comparison of the urea tolerance of 
Males

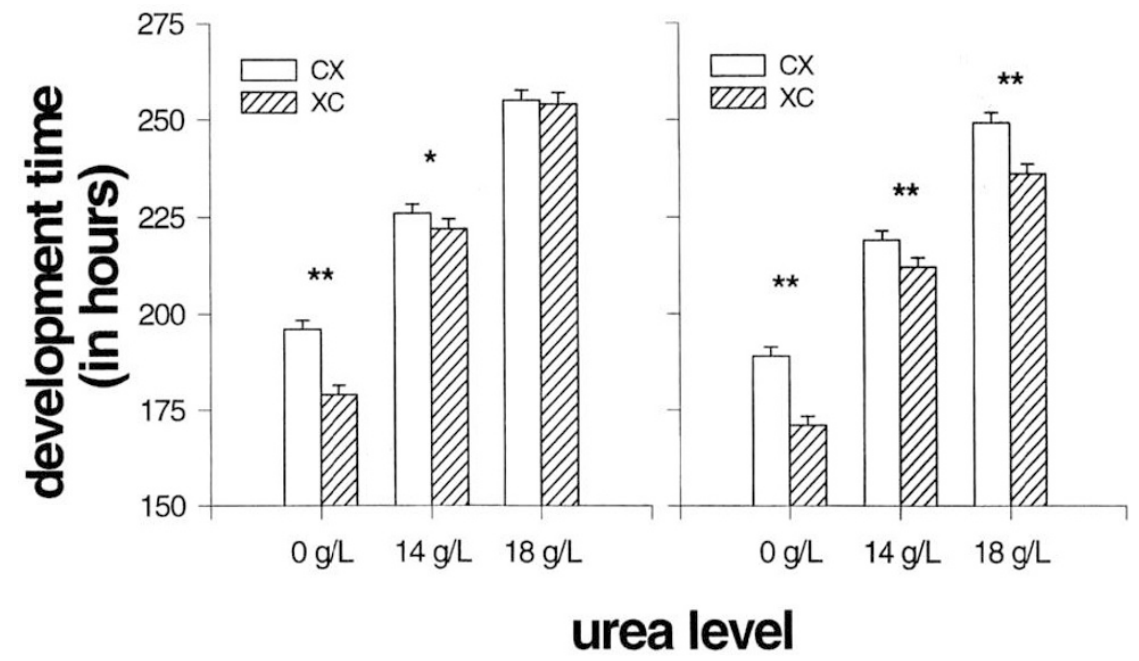

Females

Fig. 3 Egg-hatch to adult development time of males and females from the $\mathrm{CX}$ and $\mathrm{XC}$ hybrid populations of Drosophila melanogaster at three different levels of urea. Error bars represent $95 \%$ confidence intervals about the mean of five replicate populations, and were calculated using least squares estimates of the standard errors of cell means in the randomized block ANOVA $\left({ }^{*} P<0.05\right.$, $\left.{ }^{* *} P<0.01\right)$.
CX and XC hybrids with that of the parental populations provides evidence for a substantial dominance component to the genetic control of urea tolerance in the MX populations. The survivorship (Fig. 1) and development time (Fig. 2) of both the $\mathrm{CX}$ and $\mathrm{XC}$ hybrids was significantly greater than the mid-parent value in the presence of urea in the food medium. The dominance pattern also appears to be stable, at least over the two levels of urea used in this study; in studies of insecticide resistance, alleles for resistance are often seen to switch from dominance to recessiveness as the dosage increases (Georghiou \& Taylor, 1977; Taylor \& Georghiou, 1979).

The differences between the reciprocal hybrids in survivorship, and especially the different patterns of relative urea tolerance, as reflected in the effect of urea on development time, in male and female CX and $\mathrm{XC}$ hybrids (Fig. 3), provide evidence for at least partial $\mathrm{X}$-linked control of urea tolerance in the MX populations. Unfortunately, a similar comparison of sexes could not be carried out with the survivorship data because we cannot be sure that equal numbers of male and female larvae were put into the vials at the start of the assay. The significantly faster development of the XC males (at low urea levels) and females (at all urea levels used in this study), compared with their CX counterparts (Fig. 3), is consistent with a detrimental nongenetic maternal effect of MX mothers on the development time of their offspring. At higher urea levels, the difference between the $\mathrm{CX}$ and $\mathrm{XC}$ hybrids resulting from this maternal effect appears to be compensated for in the CX males by their greater urea tolerance, inherited via the $\mathrm{X}$ chromosome from their $\mathrm{MX}$ mothers. The $\mathrm{CX}$ females, not differing from $\mathrm{XC}$ females in their $\mathrm{X}$ chromosome composition, remain slower developers across all urea levels (Fig. 3).

The indication of dominance and X-linkage of larval urea tolerance in this study suggests that it is likely that there may be a relatively small number of loci exerting a major effect on the expression of this trait. Further genetic investigations will be needed to assess this possibility. Further studies on the exact form in which nitrogenous waste tends to accumulate in Drosophila cultures, urea or ammonia, as well as studies of whether urea tolerance and ammonia tolerance are, at least partially, physiologically related, will also help to achieve a more detailed understanding of larval adaptation to crowding in Drosophila.

\section{Acknowledgements}

We thank D. J. Borash, G. T. Ho, S. In, V. K. Lun, W. A. Oshiro and W. Wu for assistance in the laboratory, and J. Shiotsugu for very helpful advice on the urea tolerance assays. This study was supported by grants DEB-9410281 from the National Science Foundation and AG09970 from the National Institutes of Health to L. D. Mueller.

\section{References}

AIKEN, R. B. AND GIBO, D. L. 1979. Changes in fecundity of Drosophila melanogaster and $D$. simulans in response to selection for competitive ability. Oecologia, 43, 63-77.

BAUER, S. J. AND SOKOLOWSK1, M. B. 1985. A genetic analy- 
sis of path length and pupation height in a natural population of Drosophila melanogaster. Can. J. Genet. Cytol., 27, 334-340.

BAUER, s. J. AND SOKOLOWSKI, M. B. 1988. Autosomal and maternal effects on pupation behaviour in Drosophila melanogaster. Behav. Genet., 18, 81-97.

BOTELlA, L. M., MOYA, A. AND MENSUA, J. L. 1983. Effects of urea on viability and mean developmental time in $D$. melanogaster larvae. Drosoph. Inf. Serv., 59, 23-24.

BOTELLA, L. M., MOYA, A., GONZALEZ, M. C. AND MENSUA, J. L. 1985. Larval stop, delayed development and survival in overcrowded cultures of Drosophila melanogaster: effect of urea and uric acid. J. Insect Physiol., 31, 179-185.

BUDNIK, M. AND BRNCIC, D. 1974. Preadult competition between Drosophila pavani, D. melanogaster, D. simulans and D. willistoni. Ecology, 55, 657-661.

BUDNIK, M. AND BRNCIC, D. 1976. Effects of larval biotic residues on viability in four species of Drosophila. Evolution, 29, 777-781.

BURNET, B., SEWELL, D. AND BOS, M. 1977. Genetic analysis of larval feeding behaviour in Drosophila melanogaster. II. Growth relations and competition between selected lines. Genet. Res., 30, 149-161.

DE BELLE, J. S. AND SOKOLOWSKI, M. B. 1987. Heredity of rover/sitter: alternative foraging strategies of Drosophila melanogaster. Heredity, 59, 73-83.

DE BELLE, J. S., HILLIKER, A. J. AND SOKOLOWSKI, M. B. 1989. Genetic localization of foraging (for): a major gene for larval behaviour in Drosophila melanogaster. Genetics, 123, 157-163.

DE BELLE, J. S., SOKOLOWSKI, M. B. AND HILLIKER, A. J. 1993. Genetic analysis of the foraging microregion of Drosophila melanogaster. Genome, 36, 94-101.

DE MIRANDA, J. R. AND EGGLeston, P. 1988. Larval competition in Drosophila melanogaster. I. Estimation of larval growth parameters. Heredity, 60, 205-212.

FREEMAN, M. F. AND TUKEY, J. w. 1950. Transformations related to the angular and the square root. Ann. Math. Stat., 21, 607-611.

GEORGHIOU, G. P. AND TAYLOR, C. E. 1977. Genetic and biological influences in the evolution of insecticide resistance. J. Econ. Entomol., 70, 319-323.

GODOY-HERRERA, R. 1986. The development and genetics of digging behaviour in Drosophila melanogaster. Heredity, 56, 33-41.

GODOY-HERRERA, R. 1994. Biometrical analysis of larval digging behavior in Drosophila melanogaster. Behav. Genet., 24, 427-432.

JOSHI, A. AND MUELLER, L. D. 1988. Evolution of higher feeding rate in Drosophila due to density-dependent natural selection. Evolution, 42, 1090-1093.

JOSHI, A. AND MUELLER, L. D. 1993. Directional and stabilizing density-dependent natural selection for pupation height in Drosophila melanogaster. Evolution, 47, 176-184.

JOSHI, A. AND MUELLER, L. D. 1996. Density-dependent natural selection in Drosophila: trade-offs between larval food acquisition and utilization. Evol. Ecol. (in press).

MACARTHUR, R. M. AND WILSON, E. O. 1967. The Theory of Island Biogeography. Princeton University Press, Princeton, NJ.

MARKOW, т. A. 1979. A survey of intra- and inter-specific variation for pupation height in Drosophila. Behav. Genet., 9, 209-217.

MUELLER, L. D. 1990. Density-dependent natural selection does not increase efficiency. Evol. Ecol., 4, 290-297.

MUELLER, L. D. 1995. Adaptation and density-dependent natural selection. In: Levine, L. (ed.) Genetics of Natural Populations: the Continuing Importance of Theodosius Dobzhansky, pp. 222-238. Columbia University Press, New York.

MUELLER, L. D. AND SWEET, V. F. 1986. Density-dependent natural selection in Drosophila: evolution of pupation height. Evolution, 40, 1354-1356

MUELLER, L. D., GUO, P. Z. AND AYALA, F. J. 1991. Densitydependent natural selection and trade-offs in lifehistory traits. Science, 253, 433-435.

MUELLER, L. D., GRAVES, J. L. AND ROSE, M. R. 1993. Interactions between density-dependent and age-specific selection in Drosophila melanogaster. Funct. Ecol., 7, 469-479.

PEREIRA, H. S., MACDONALD, D. E., HILLIKER, A. J. AND soKolowski, M. B. 1995. Chaser (Csr), a new gene affecting larval foraging behavior in Drosophila melanogaster. Genetics, 141, 263-270.

ROSE, M. R. 1984. Laboratory evolution of postponed senescence in Drosophila melanogaster. Evolution, 38, 1004-1010.

SAMEOTO, D. D. AND MILLER, R. s. 1968. Selection of pupation site by Drosophila melanogaster and D. simulans. Ecology, 49, 177-180.

SEWELL, D., BURNET, B. AND CONNOLLY, K. 1975. Genetic analysis of feeding behaviour in Drosophila melanogaster. Genet. Res., 24, 163-173.

sOKOLOWSKI, M. B. 1985. Ecology, genetics and behavior of Drosophila larval foraging and pupation behavior. $J$. Insect Physiol., 31, 857-864.

TAYLOR, C. E. AND GEORGHIOU, G. P. 1979. Suppression of insecticide resistance by alteration of gene dominance and migration. J. Econ. Entomol., 72, 105-109. 